\title{
Appropriately Tuning Stochastic- \\ Psychometric Properties of the Balloon \\ Analogue Response Task (BART)
}

Di Plinio, $\mathrm{S}^{1, *}$, Pettorruso, $\mathrm{M}^{1}$, Ebisch, $\mathrm{SJH}^{1,2}$

1 Department of Neuroscience, Imaging, and Clinical Sciences, G D'Annunzio University of Chieti-Pescara, Chieti, Italy

2 Institute for Advanced Biomedical Technologies (ITAB), G D'Annunzio University of Chieti-Pescara, Chieti, Italy

* Corresponding author: simone.diplinio@unich.it

Running title: Stochastic variants of the BART

Keywords: BART; Psychometrics; Task optimization; Risk-taking; Computational Neuroscience; Stochasticity

\begin{abstract}
The Balloon Analogue Risk Task (BART) allows to experimentally assess individuals' risk-taking profiles in an ecologically sound setting. Many psychological and neuroscientific studies implemented the BART for its simplicity and intuitive nature. However, some issues in the design of the BART are systematically unconsidered in experimental paradigms, which may bias the estimation of individual risk-taking profiles. Since there are no methodological guidelines for implementing the BART, many variables (e.g., the maximum explosion probabilities, the rationale underlying stochastic events) vary inconstantly across experiments, possibly producing contrasting results. Moreover, the standard version of the BART is affected by the interaction of an individual-dependent, unavoidable source of stochasticity with a trial-dependent, more ambiguous source of stochasticity (i.e., the probability of the balloon to explode). This paper shows the most appropriate experimental choices for having the lowest error in the approximation of risk-taking profiles. Performance tests of a series of simulated data suggest that a more controlled, eventually nonstochastic version of the BART, better approximates original risk-taking profiles. Selecting optimal BART parameters is particularly important in neuroscience experiments to optimize the number of trials in a time window appropriate for acquiring neuroimaging data. We also provide helpful suggestions to researchers in many fields to allow the implementation of optimized risk-taking experiments using the BART.
\end{abstract}




\section{INTRODUCTION}

The administration of psychometric tests is a crucial phase in scientific investigations aimed at understanding human behavior, including cognitive and behavioral individual differences, predispositions toward clinical and subclinical symptoms, and the neurocognitive framework supporting psychometric variability (Lenhard et al., 2019; Zickar, 2020; Tonini et al., 2021). To achieve such aims, it is crucial that the statistical-mathematical structure and the psychological-philosophical background of psychometric instruments are sound, unbiased, and consistent (Nichols et al., 2017; Steiner and Frey, 2022). Ideally, reproducible simulations should test task performance variations for their psychometric and stochastic properties, including their general efficiency in measuring latent behavior (Schonberg et al., 2011; Bajracharya and Duboz, 2013; Donkin et al., 2017; Fitzpatrick, 2019).

The Balloon Analogue Response Task (BART) is a valuable laboratory-based psychometric instrument to assess risk-taking behavior in healthy and clinical populations. From its initial development (Lejuez et al., 2002), the BART has been used in many experiments, producing a large body of exciting literature. The widespread utilization of the BART is motivated by its capability in recreating a natural ecological experience (Lejuez et al., 2002, 2003, 2005; De Groot, 2020) to uncover (neuro)cognitive underpinnings of risk-taking. Measurements of risk-taking behavior are interesting for both general and clinical research. In fact, such trait as indexed by BART scores has been associated with dysfunctional psychophysiological phenotypes, including anxiety (Maner et al., 2007; Buelow and Barnhart, 2017), clinical disorders (Hunt et al., 2005; Swogger et al., 2010; Dominguez et al., 2011; Cheng et al., 2012; Robbins et al., 2012; Reddy et al., 2014; Fischer et al., 2015; Brown et al., 2015; Luk et al., 2019; Tikasz et al., 2019; Boka et al., 2020), abuse of heavy drugs (Hopko et al., 2006; Vassileva and Conrod, 2019), smoking attitudes (Lejuez et al., 2003; Lejuez et al., 2005; Dean et al., 2011; Hanson et al., 2014), alcohol consumption and related symptoms (Skeel et al., 2008; Fernie et al., 2010; Ashenhurst et al. 2011; Weafer et al., 2011; DeMartini et al. 2014; King et al., 2014), gambling (Holt et al., 2003; Mishra et al., 2017), risky sexual behavior (Lejuez et al., 2004; Bornovalova et al., 2008; Lawyer, 2013), and even with pandemic-related behavior (Guenther et al., 2021; for reviews on risk-taking and related dysfunctions see: Leigh, 1999; Turner et al., 2004; Isles et al., 2019). Moreover, BART use has been suggested as a potential marker for dissecting disease-related endophenotypes (Long et al., 2020).

The experimental design of the BART is quite intuitive and straightforward (Lejuez et al., 2002). In every trial, a balloon is presented, and the participant is asked to either take an award proportional to the current size of the balloon or attempt inflating the balloon to increase the value of the award. Every time the participant chooses to inflate the balloon, it can be inflated or explode. The explosion of the balloon implies that the award for the current trial is zero (or a negative value). For its simplicity and ecological validity, the BART has also been used in neuroimaging studies (Rao et al., 2008; Cazzell et al., 2012; Sela et al., 2012; Xu et al., 2016; Guo et al., 2018; De Groot and Strien, 2019) and showed reliability for both behavioral and neural evoked responses (White et al., 2008; Li et al., 2020).

Although the underlying paradigm is reasonably practical and straightforward, the BART in its standard design entails unsolved criticalities that may hinder the usefulness of the collected data. Previous works discussed putative issues of the BART, including the censoring of information, the confusion of risk with the expected value, a poor distinguishing between uncertainty and risk, and the ambiguity in the definition of adaptive versus maladaptive behavior (Schonberg et al., 2011; De Groot and Thurik, 2018; De Groot, 2020; Canning et al., 2022). While these concerns are relevant, a significant source of inaccuracies in the BART is the unsafe interaction between two stochastic processes. First, individual uncertainty and noisy behavior are non-avoidable sources of stochasticity in reconstructing individual profiles (although this stochasticity is a valuable source of information). Second, implementing random consequences of individual choices (inflating versus explosion) means decreasing experimental control over the paradigm 
and introducing an additional source of stochasticity. The impact of this second source of stochasticity has not been appropriately investigated yet.

In this study, we show that the power of the BART to reconstruct individual risk-taking profiles significantly raises if its methodological implementation is appropriately controlled. More specifically, controllable sources of stochasticity must be handled to achieve accurate experiments. To propose an unbiased, reproducible, and controlled version of the BART, we implement a series of simulations with varying parameters (i.e., explosion probability functions, virtual risk-taking profiles, number of inflations, number of trials, number of subjects, noise). These methodological improvements will be broadly relevant in the field of risk-taking psychometric measurements. They will mainly assist in situations in which researchers must improve their experimental paradigm due to time and instrumental limitations, like, for example, in the case of clinical neuroimaging experiments. The evidence reported by our investigation, together with the implementation of naturalistic and unbiased measures (Schmitz et al., 2016; Yakobi and Danckert, 2021; Coon and Lee, 2021; Steiner and Frey, 2022), will allow scientists to adopt the BART in the best possible way following their experimental demand.

\section{METHODS}

The double-stochasticity in the BART arises from the interaction of the individual-dependent stochasticity and the design-dependent stochasticity. The present study explicitly addresses the design-dependent stochasticity in the BART. Although precise methodological details about the experimental design are sometimes lacking, the great majority of studies implementing the BART linearly or exponentially modulate the probability of balloon explosion across consecutive inflations. Generally, the explosion probability is modulated to increase within 0 ( $0 \%$ explosion, the balloon will surely inflate) and 1 (100\% explosion, the balloon will explode).

Modeling explosions in multiple variants of the BART. Conceptually, the only difference among the various simulations we implemented was how explosion probabilities were modeled. We incorporated and expanded the variations used in past studies utilizing the BART to investigate the impact of the following parameters in the reconstruction of actual risk-taking profiles: the explosion probability maximum threshold; the function used to calculate monotonically increasing explosion probabilities across inflations; the number of inflation events $\&$ the number of trials; the number of virtual players (participants); the noise in the virtual players' behavior; the implementation of non-stochastic (i.e., deterministic) explosions.

Simulating virtual risk-taking profiles. We tested the performance of different BART versions using a series of simulations implemented in MatLab (The Mathworks, version 2019b). In these simulations, several risk-taking profiles were initially generated. We used two sets of risk-taking profiles for the simulations: the first set comprehended monotonically decreasing functions with a non-linear decay; the second set comprehended monotonically decreasing functions generated using random numbers. These simulated profiles were chosen since they are realistically able to approximate actual "ordered/stable" and "noisy" risk-taking profiles reported and discussed in the BART literature (Schonberg et al., 2011; Yakobi and Danckert, 2021). Assuming N virtual subjects and T trials per subject, the simulation of risk-taking profiles ultimately produced, for each virtual subject $n$, and for each trial $t$, a series of $\mathrm{K}$ monotonically decreasing numbers comprised between 100 and 0 (where $100=$ the virtual subject will surely try to inflate the balloon at this inflation event, and $0=$ the virtual subject will surely choose the award). The parameters $\mathrm{K}, \mathrm{N}$, and $\mathrm{T}$ were varied according to different parameters (see below). These profiles are shown in Figure 1A-B.

Simulating decision matrices. After generating individual risk-taking profiles, the balloon analogue risk task procedure was simulated for each subject, using a maximum of $\mathrm{K}$ inflation events. Before the actual simulation of the BART, an individual decision matrix was produced as follows. In each trial, a 
decision of the $\mathrm{n}^{\text {th }}$ virtual subject is simulated by extracting a random number between 1 and 100: if the random number is above the critical threshold for that $\mathrm{n}^{\text {th }}$ subject, in the $\mathrm{t}^{\text {th }}$ trial, and in the $\mathrm{k}^{\text {th }}$ inflation event, then the corresponding decision for the current trial is set to "take the award"; alternatively, if the random number is below the critical threshold for that $\mathrm{n}^{\text {th }}$ subject in the $k^{\text {th }}$ inflation event, then the corresponding decision for the current trial is set to "try inflating the balloon". Importantly, modeling exactly the same degree of intra-subject variability (i.e., subject-related stochasticity) is mandatory to investigate the accuracies of different parametrizations of the BART. Thus, the decision matrix was simulated for each virtual subject at each simulation cycle before running the BART itself. In this way, a Tby-K decision matrix was produced from each virtual subject (i.e., from each risk-taking profile). To note, this stage entailed the first, unavoidable source of stochasticity in the BART.

The BART. The individual decision matrix was subsequently applied to the task. In each trial, the virtual decision is used to prompt the BART algorithm alternatively toward the "take the award" or "try inflating the balloon" choice. The "take award" option leads to obtaining a certain amount of money and going to the subsequent trial. Instead, the "try inflating the balloon" option led to the extraction of a random number between 1 and 100: if the random number was below the critical explosion threshold in the $k^{\text {th }}$ inflation event, in the $t^{\text {th }}$ trial, then the balloon exploded; alternatively, if the random number is equal or above the threshold, then the trial continues to the next inflation $\left(k^{\text {th }}+1\right)$. This stage was modeled for three explosion probability functions: linear increase function, exponential increase function, and logarithmic increase function. Moreover, three maximum explosion thresholds were set: $50 \%, 75 \%$, and $100 \%$. These functions are represented in Figure 1C. To note, this stage represented the second source of stochasticity in the BART.

Simulation parameters. Investigating an increasing number of maximum inflation events $(K)$ and trials $(T)$ is crucial since it influences the psychometric properties of the BART (Lejuez et al., 2002; Fecteau et al., 2007; Rao et al., 2008). Furthermore, many neuroimaging experiments must comply with time and instrumental limitations, utilizing fewer trials in event-related designs. We selected a range of combinations of $K$ and $T$ to allow a comprehensive examination of the effect of double sources of stochasticity in the BART. Thus, $K$ was tuned to have the values [ 6101624 48]. At the same time, the number of trials was varied systematically to investigate both scenarios with time limitations (e.g., neuroimaging studies) and less coerced procedures (e.g., behavioral studies). Expressly, with a low number of inflations (K=6, K=10, $K=16)$, the total number of trials $T$ was set to 48 . Instead, with a high number of inflations $(K=24, K=48), T$ was set to 150 . We did not expect the number of subjects $\mathrm{N}$ to impact errors particularly. However, given the importance of combining trial number and sample size in neuroscience (Nichols et al., 2017; Chen et al., 2022), we investigated variable sample sizes in the current study, ranging from 20 to 100 virtual subjects $[20,50,100]$. Finally, we investigated two levels of noise in the virtual subjects: in the low-noise condition, each individual decision of the decision matrix was distorted by adding a random number in the range [-.1 .1]; instead, in the high-noise condition, each individual decision of the decision matrix was distorted by adding a random number in the range [-.4 .4].

Non-stochastic BART. In the standard, stochastic BART (s-BART), each time the virtual participant attempted to inflate the balloon, it alternatively exploded or not depending on a random number. Instead, in the deterministic BART (d-BART), balloons were programmed to explode from a particular inflation event without the need for further unavoidable randomizations. In the d-BART, when the virtual subject attempted inflating the balloon (i.e., when the $k^{\text {th }}$-by- $t^{\text {th }}$ element of the decision matrix was "try inflating"), it deterministically exploded or not based on the maximum number of inflations allowed for that balloon: if the max number of inflations was exceeded, then the balloon exploded; if not, then the trial continued to the next inflation event. We also implemented this d-BART in the simulations since it may be presumed that avoiding the second source of stochasticity may improve the estimation of risk-taking profiles. To note, in both the s-BART and the d-BART, subjective priors would be equivalent. 
Statistics. Reconstructed profiles were averaged and plotted separately for each parametrization. The performance was assessed using linear mixed-effects regression models. The dependent variable was the estimation error (the difference between the real risk-taking profile and the reconstructed profile). Fixed effects were added for the factor threshold (three levels: 50\%, 75\%, 100\%) and the factor function (three levels: linear, exponential, logarithmic). Random intercepts were added for each virtual subject, and random intercepts and slopes were added concerning the grouping factor inflation. Thus, the model was in the Wilkinson form "error threshold * function + (1 / subject) + (threshold + function / inflation)". Secondly, we compared the best combination of threshold \& function from the s-BART with the d-BART. The linear mixed-effects models were applied independently for each combination of trials, inflations, and noise levels. Linear contrasts within the model were used to directly compare appropriate levels of the fixed factors.

\section{RESULTS}

We ran each simulation scenario (i.e., each unique combination of parameters) 100 times separately for low and high noise levels. The total computation time was approximately 50 hours on a computer with an $11^{\text {th }}$ generation i7 quad-core processor $(2.80 \mathrm{GHz})$ and $16 \mathrm{~GB}$ of installed RAM. After each cycle, original risktaking profiles were reconstructed for each virtual subject using each combination of parameters for modeling explosions (linear increase with $100 \%$ maximum threshold, linear increase with $75 \%$ maximum threshold, linear increase with 50\% maximum threshold, exponential increase with $100 \%$ maximum threshold, exponential increase with $75 \%$ maximum threshold, exponential increase with $50 \%$ maximum threshold, logarithmic increase with $100 \%$ maximum threshold, logarithmic increase with $75 \%$ maximum threshold, logarithmic increase with 50\% maximum threshold, deterministic BART).

Figure $2 \mathrm{~A}$ shows the performance of different parameter combinations with low levels of noise. The fixed factors and the interaction were significant in almost every situation, indicating an effect of both threshold and function used to model balloon explosion on the error in estimating original risk-taking profiles. More specifically, with 6 maximum inflations $(\mathrm{K}=6)$, we found a trend for threshold $\left(\mathrm{F}_{\mathrm{THR}}=2.6\right.$, $\left.\mathrm{p}_{\text {THR }}=.07\right)$, a non-significant effect for function ( $\left.\mathrm{F}_{\mathrm{FUN}}=0.7, \mathrm{p}_{\mathrm{FUN}}=.49\right)$, and a significant interaction thresholdby-function ( $\left.F_{\mathrm{INT}}=775.7, \mathrm{p}_{\mathrm{INT}}<.001\right)$. With higher $\mathrm{K}$ values, these effects were always significant $(\mathrm{K}=10$ : $\mathrm{F}_{\mathrm{THR}}=7.2, \mathrm{p}_{\mathrm{THR}}<.001, \mathrm{~F}_{\mathrm{FUN}}=3.2, \mathrm{p}_{\mathrm{FUN}}=.04, \mathrm{~F}_{\mathrm{INT}}=974.2, \mathrm{p}_{\mathrm{INT}}<.001 ; \mathrm{K}=16: \mathrm{F}_{\mathrm{THR}}=14.4, \mathrm{p}_{\mathrm{THR}}<.001, \mathrm{~F}_{\mathrm{FUN}}=5.4, \mathrm{p}_{\mathrm{FUN}}=.004$, $\mathrm{F}_{\mathrm{INT}}=1104.6, \mathrm{p}_{\mathrm{INT}}<.001 ; \mathrm{K}=24: \mathrm{F}_{\mathrm{THR}}=24.5, \mathrm{p}_{\mathrm{THR}}<.001, \mathrm{~F}_{\mathrm{FUN}}=7.5, \mathrm{p}_{\mathrm{FUN}}<.001, \mathrm{~F}_{\mathrm{INT}}=2044.2, \mathrm{p}_{\mathrm{INT}}<.001 ; \mathrm{K}=48$ :

$\left.\mathrm{F}_{\mathrm{THR}}=39.0, \mathrm{p}_{\mathrm{THR}}<.001, \mathrm{~F}_{\mathrm{FUN}}=11.3, \mathrm{p}_{\mathrm{FUN}}<.001, \mathrm{~F}_{\mathrm{INT}}=2106.4, \mathrm{p}_{\mathrm{INT}}<.001\right)$. Linear contrasts of interest for the effects of interest are reported in Table I (center columns). The performance of different parameter combinations with high levels of noise are shown in Figure 2B. Also in this case, the fixed factors and the interaction effects were significant. More specifically, with 6 maximum inflations $(K=6)$, we found a significant effect of threshold ( $\left.F_{T H R}=3.5, p_{T H R}=.03\right)$, a non-significant effect for function ( $\left.F_{F U N}=0.7, p_{F U N}=.47\right)$, and a significant interaction threshold-by-function ( $\left.F_{\mathrm{INT}}=596.3, \mathrm{p}_{\mathrm{INT}}<.001\right)$. With 10 maximum inflations $(K=10)$, we found a trend for threshold ( $\left.F_{\text {THR }}=7.6, p_{\text {THR }}<.001\right)$, a trend for function ( $\left.F_{\mathrm{FUN}}=3.0, \mathrm{p}_{\mathrm{FUN}}=.05\right)$, and a significant interaction threshold-by-function $\left(\mathrm{F}_{\mathrm{INT}}=814.3, \mathrm{p}_{\mathrm{INT}}<.001\right)$. With higher $\mathrm{Ks}$, effects were always significant $\left(\mathrm{K}=16: \mathrm{F}_{\mathrm{THR}}=11.1, \mathrm{p}_{\mathrm{THR}}<.001, \mathrm{~F}_{\mathrm{FUN}}=3.5, \mathrm{p}_{\mathrm{FUN}}=.03, \mathrm{~F}_{\mathrm{INT}}=885.8, \mathrm{p}_{\mathrm{INT}}<.001 ; \mathrm{K}=24: \mathrm{F}_{\mathrm{THR}}=20.9, \mathrm{p}_{\mathrm{THR}}<.001, \mathrm{~F}_{\mathrm{FUN}}=7.8\right.$, $\left.\mathrm{p}_{\mathrm{FUN}}<.001, \mathrm{~F}_{\mathrm{INT}}=1750.3, \mathrm{p}_{\mathrm{INT}}<.001 ; \mathrm{K}=48: \mathrm{F}_{\mathrm{THR}}=30.8, \mathrm{p}_{\mathrm{THR}}<.001, \mathrm{~F}_{\mathrm{FUN}}=10.2, \mathrm{p}_{\mathrm{FUN}}<.001, \mathrm{~F}_{\mathrm{INT}}=1397.9, \mathrm{p}_{\mathrm{INT}}<.001\right)$. Linear contrasts of interest for the effects of interest are reported in Table I (right columns).

Results related to the comparison of the s-BART versus d-BART are shown in Figure $3 A$ (low noise levels) and 3B (high noise levels). For simplicity, we reported the comparison between the best s-BART model (i.e., 50\% threshold and exponential explosion probability) and the d-BART model. Although the differences are smaller than in the previous models, it can be observed that the s-BART slightly outperforms the d-BART. With $\mathrm{K}=6$, the effect was not significant neither with low $(F=2.4, p=.12)$ or high 
noise $(\mathrm{F}=3.4, \mathrm{p}=.06)$. Instead, with higher number of inflations, the stochastic exponential and low threshold (50\%) BART always outperformed the deterministic BART with both low noise levels ( $K=10: F=7.6$, $p=.006 ; K=16: F 11.9=, p<.001 ; K=24: F=14.4, p<.001 . ; K=48: F=9.3, p=.002)$ and high noise levels $(K=10:$ $F=9.6, p=.002 ; K=16: F 10.7=, p=.001 ; K=24: F=18.4, p<.001 . ; K=48: F=6.8, p=.009)$.

These results demonstrate that both the threshold and the function used to model stochastic explosions in the BART affect the performance in approximating original risk-taking profiles, independently on the noise level, maximum number of inflation events, and number of trials. Moreover, a crucial effect of the noise levels on the risk-taking profile estimation is appreciable: higher noise levels impact the approximation of information relative to the risk-taking profiles in earlier inflation events (first half), while the difference between high-noise and low-noise simulations is reduced in later inflations (second half). Finally, we show that the stochastic (standard) modeling of explosion probabilities is better than the deterministic modeling. The results reported here entail simulations with players' virtual risk-taking profiles modeled using monotonically decreasing functions generated using random numbers, with $\mathrm{N}=50$. However, such effects are equivalent when varying the number of players ( $\mathrm{N}=20$ and $\mathrm{N}=100)$ and using monotonically decreasing functions with an exponential decay to model virtual players' risk-taking profiles. These results are shown in the supplementary file.

\section{DISCUSSION}

Our study investigates a theoretical-methodological issue in one of the most frequently employed psychometric instruments to assess the risk propensity of individuals: the Balloon Analogue Risk Task (BART, Lejuez, 2002). We investigated the relationship between forms of stochasticity and expected psychometric measurements in experiments involving the BART. Two types of stochasticity coexist in this task: the first one reflects an informative variability related to individual uncertainty and noise (De Groot and Thurik, 2018; Yakobi and Danckert, 2021; De Groot, 2020); the second one arises when the experimental design is not appropriately controlled and theoretically does not convey any helpful information on risk-taking profiles.

We demonstrate that controlling the stochastic trial parametrization in the BART leads to a better approximation of the original risk-taking profiles. In particular, we show that using exponential functions to model explosion probabilities reduces estimation errors up to $9-10 \%$ for the original virtual risk-taking profiles. Moreover, using lower thresholds (maximum probability of explosions) reduces estimation errors up to $4-5 \%$. Interaction effects in our models demonstrate that lower maximum thresholds of explosion probabilities (50\% and $75 \%$ ) combined with exponential functions for modelling stochastic explosions are the best combination to allow an efficient estimation of true risk-taking profiles. Importantly, we show that these results are consistent across many prior profiles (virtual participants in our simulations) and across noise levels used to "bias" the virtual participants' decisions. Finally, we demonstrate that the optimized version of the stochastic BART (s-BART) has a similar performance with the non-stochastic, deterministic version of the BART (d-BART).

By implementing suboptimal designs in previous studies, the approximation of individual risk-taking behavior may have been biased or noisy (see also Kilıç et al., 2020; Yakobi and Danckert, 2021).

Comprehensive studies tend to indicate discrepancies across patterns of results involving the BART (Bishara et al., 2009; Purcell et al., 2017; De Groot and Thurik, 2018; Canning et al., 2022). For example, Maner and colleagues (2007) reported effects of anxiety on risk-taking, while a later study did not find such association (Buelow and Barnhart, 2017). King and colleagues (2014) reviewed the associations between impulsivity and alcohol consumption and observed discrepancies among studies that involved the BART. Some studies showed the BART to predict impulsivity related to alcohol abuse (Fernie et al., 2010; Rose et al., 2014) or to 
be unrelated with impulsivity (Skeel et al., 2008; Rieser et al., 2019). Moreover, DeMartini et al. (2014) found BART scores to correlate positively with the quantity of alcohol consumption but negatively with the frequency of alcohol consumption, while Ashenhurst et al. (2011) found that higher BART scores negatively correlate with alcohol-related symptoms but are unrelated to alcohol use. The literature about risky decision-making in clinical conditions is hard to interpret considering associations with BART scores: whereas some studies reported abnormal behavioral BART scores in people with schizophrenia (Boka et al., 2020; Brown et al., 2015; Dominguez et al., 2011; Reddy et al., 2014), other studies did not reproduce such results (Fischer et al., 2015; Luk et al., 2019; Tikasz et al., 2019). At the same time, studies demonstrated both positive (Reddy et al., 2014) and negative (Dominguez et al., 2011) associations with symptom severity, while another study did not find associations (Cheng et al., 2012). Given these inconsistencies, we suggest that employing the most rigorous task version (optimized s-BART or d-BART in our experiment) is recommended.

A limitation of the present study is that we did not simulate the influence of expected return on the risk-taking dynamics (Schonberg et al., 2011; De Groot and Thurik, 2018; De Groot, 2020; Canning et al., 2022). In other words, our results can be interpreted as stemming from individuals with no differences regarding the impact of the expected win on risk-taking behavior. However, our results indicating that exponential functions and lower maximum thresholds for balloon explosion provide a better estimation of behavioral profiles are presumably independent of the repercussions of expected values on behavior. Thus, notwithstanding behavioral variability and priors, our findings hold as demonstrated by our investigation of multiple simulated risk-taking profiles. A further limitation may be the utilisation of fixed thresholds instead of continuous ones. However, modelling threshold as a fixed factor with three levels $(50 \%, 75 \%, 100 \%)$ has the benefit of avoiding biases related to possible non-linear effects of the threshold. It is also helpful to mention that the non-stochastic $d$-BART implies a more stringent selection of the number of trials compared to the s-BART. When selecting the number of trials (T) for the d-BART, T must always be a product of the maximum number of inflations. For example, with 8 maximum inflation events, the total number of trials is 64 , with each class of explosion repeated 6 times $(8 * 6=64)$.

Since researchers can manipulate numerous facets of each experimental paradigm, even simple experimental procedures may have infinite applicative variants. However, choosing the proper experimental parameters is not always straightforward and intuitive. Researchers in all disciplines, including cognitive scientists, psychologists, and neuroscientists, should confidently select the most appropriate and unbiased experimental settings to generate reliable data and consequently make rational and safe claims (Schonberg et al., 2011; Bajracharya and Duboz, 2013; Donkin et al., 2017; Fitzpatrick, 2019; Chen et al., 2022). Our findings show how to optimize the reconstruction of original risk-taking profiles by allowing the extraction of the optimal amount of information through the administration of the BART. According to our findings, we suggest to model explosion probabilities using exponential monotonic increases and using a threshold (maximum explosion probability) between $50 \%$ and $75 \%$. By limiting stochasticity unrelated with subject-specific information, our findings are particularly meaningful for the implementation of the BART in neuroimaging studies and for the investigation of clinical and subclinical phenotypes. Future clinical research will benefit from the improvement of this psychometric instrument for detecting aberrant decision-making processes, thus allowing to accurately monitor the efficacy of treatment targeting the pathophysiology of risky behaviours (Pettorruso et al., 2021). Moreover, a consistent extraction of neurocognitive profiles will hopefully help guide clinicians in the selection of more personalized interventions. Researchers interested in applying this useful psychometric instrument to study risky behavior in both healthy and clinical population will greatly benefit from this design. 


\section{AUTHOR'S AVAILABILITY STATEAMENT}

Following to Open Science principles, the code used for the simulations can be obtained upon request to the Corresponding Author after publication. 

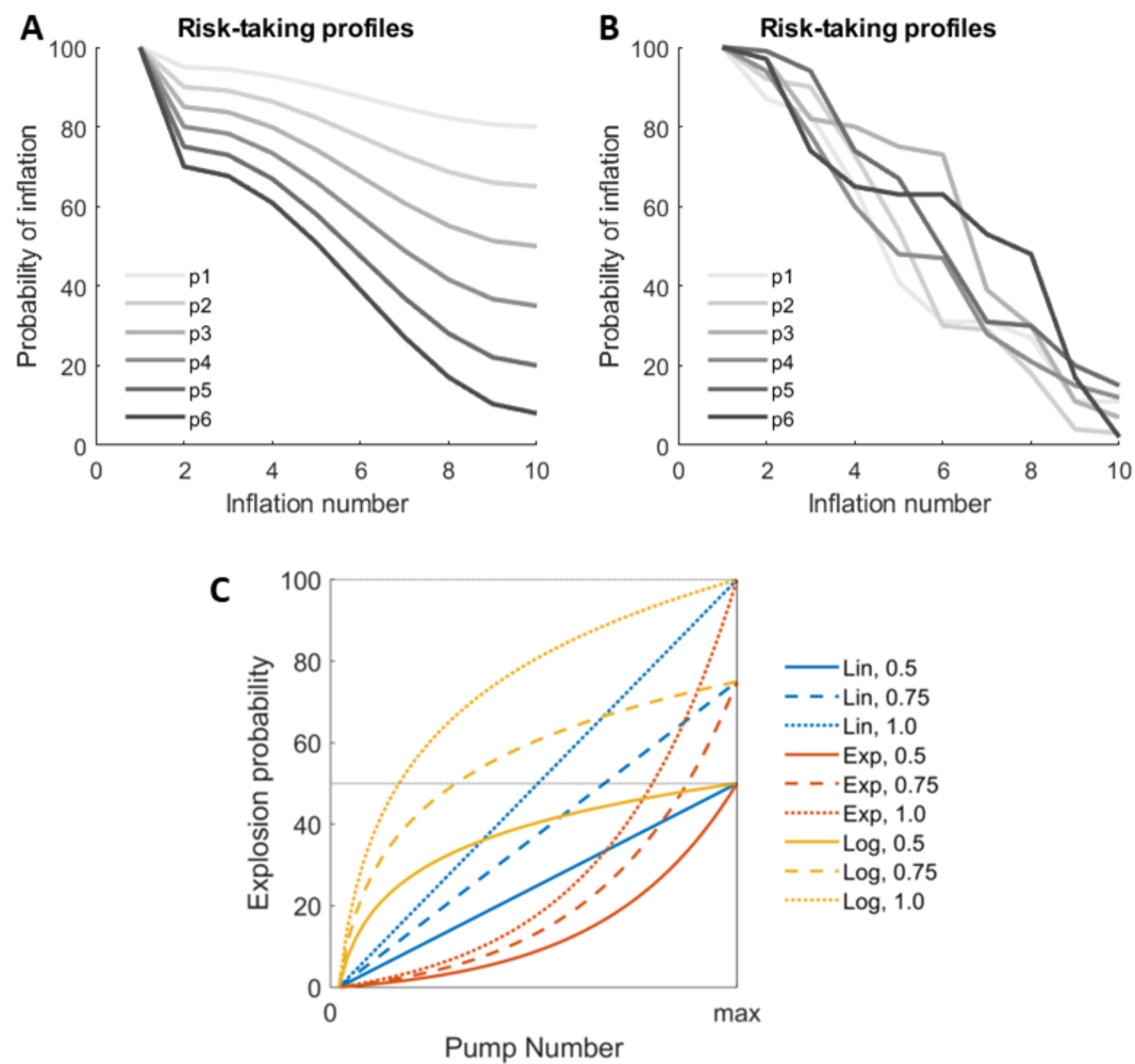

Figure 1. Parameters for the simulation. (A) Examples of virtual players with non-linear decrease in the risktaking profile. (B) Examples of virtual players with random decrease in the risk-taking profile. (C) Combination of parameters used to model explosions in the different versions of the stochastic BART analyzed. The three functions used to model explosion probabilities in consecutive inflations are coded by colors (linear, exponential, logarithmic). The three thresholds indicating maximum explosion probabilities are coded by the line type $(50 \%, 75 \%, 100 \%)$. 

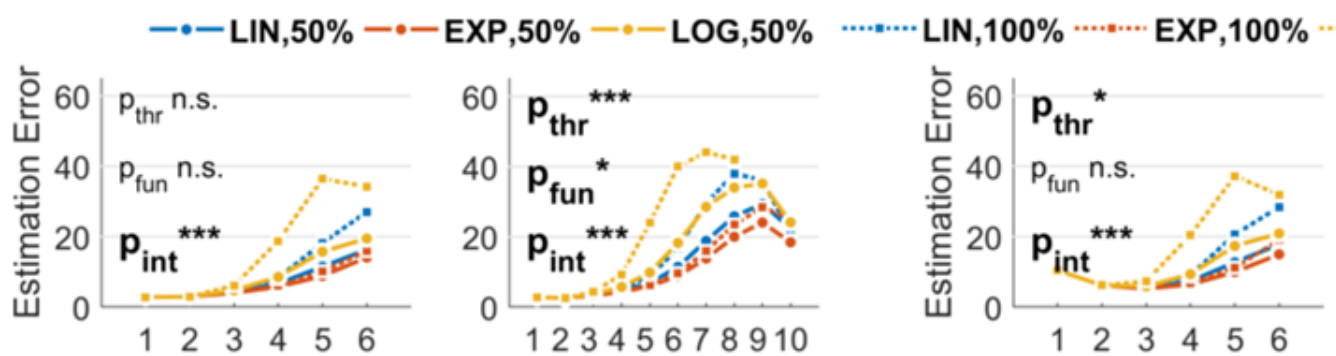

LOG, $100 \%$
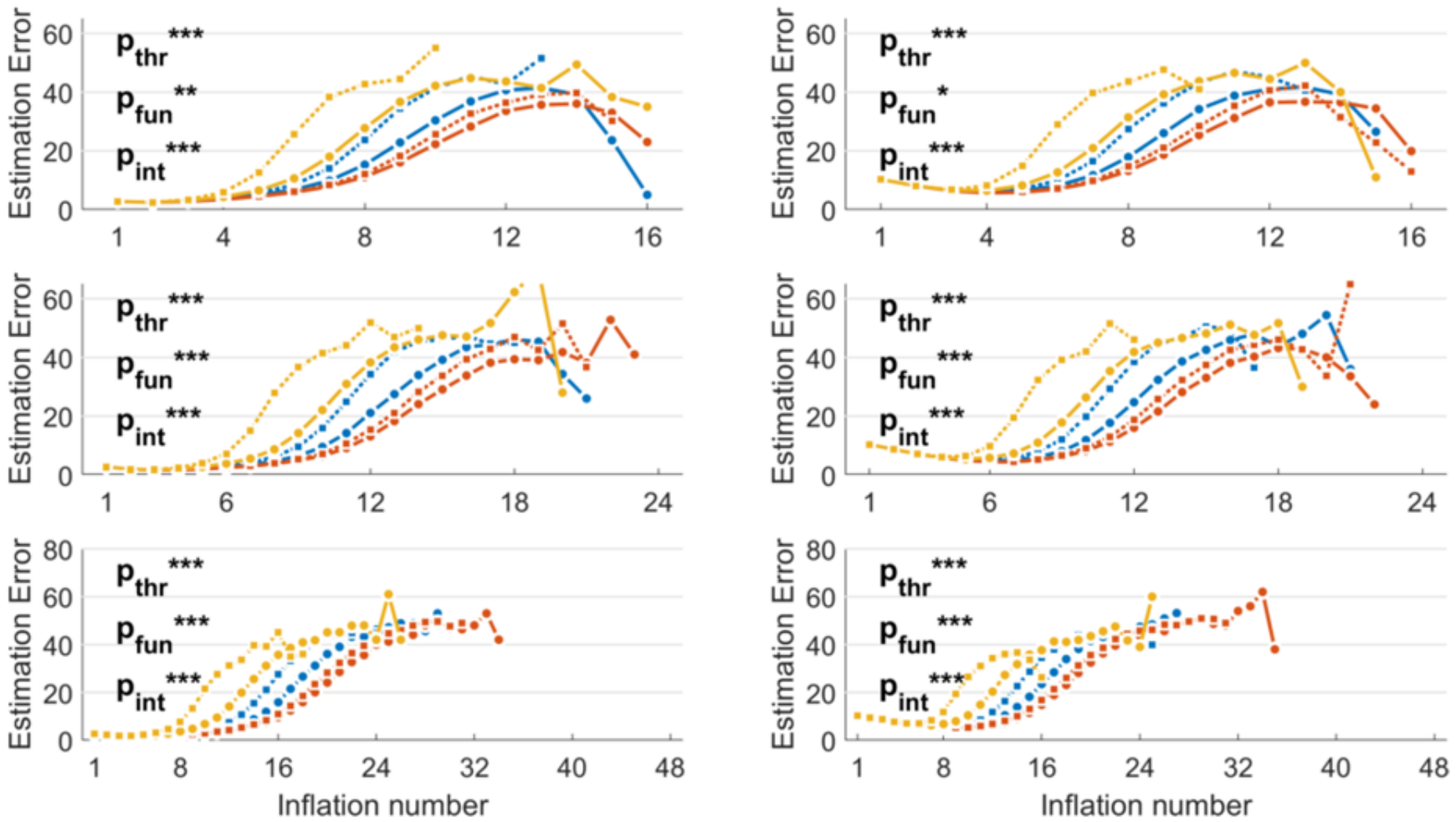

Figure 2. Estimation errors of the stochastic version of the BART implemented in the simulations. The three functions used to model explosion probabilities in consecutive inflations are coded by colors (linear, exponential, logarithmic). The three thresholds indicating maximum explosion probabilities are coded by the marker and line type (only $50 \%$ and $100 \%$ thresholds are shown to improve readability). (A) Results with low noise levels in the virtual participants' decisions. (B) Results with high noise levels in the virtual participants' decisions. Legend: ${ }^{* *}: p<.001 ;{ }^{* *}: p<.01 ;^{*}: p<.05$. 

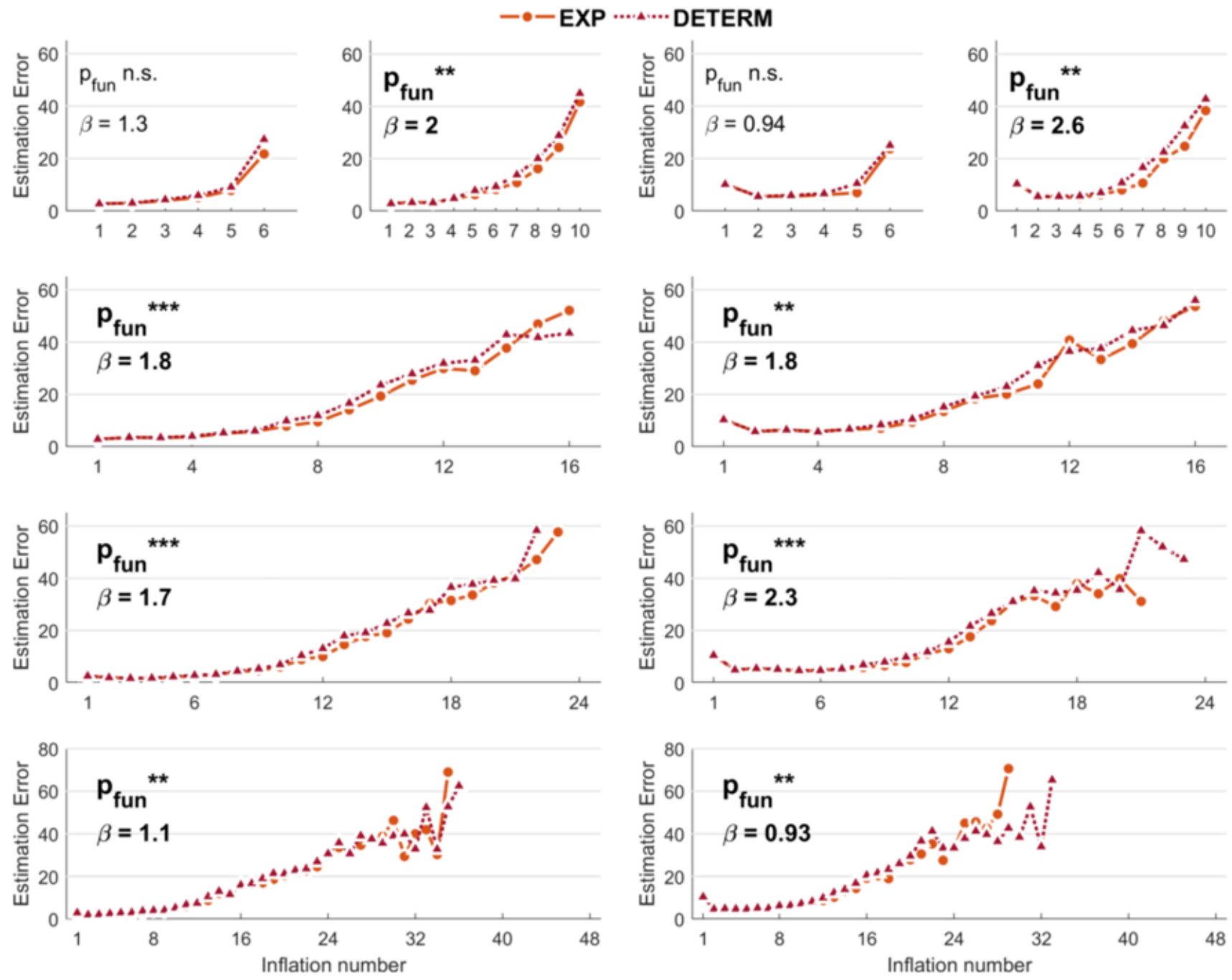

Figure 3. Estimation errors of the stochastic versus deterministic BART implemented in the simulations. With respect to stochastic BART, only results relative to explosions modelled with exponential function and $50 \%$ threshold are shown for comparison. (A) Results with low noise levels in the virtual participants' decisions. (B) Results with high noise levels in the virtual participants' decisions. Legend: ${ }^{* * *}: \mathrm{p}<.001 ;{ }^{* *}$ : $p<.01 ; *$ p $<.05$. 


\begin{tabular}{|c|c|c|c|c|c|c|}
\hline \multirow{2}{*}{$\begin{array}{c}\text { Inflations } \\
\text { (trials) }\end{array}$} & \multirow{2}{*}{ Factor } & \multirow{2}{*}{$\begin{array}{c}\text { Linear contrast } \\
\text { of interest }\end{array}$} & \multicolumn{2}{|c|}{ Low Noise } & \multicolumn{2}{|c|}{ High Noise } \\
\hline & & & $\beta$ (SE) & $P$ value & $\beta$ (SE) & $P$ value \\
\hline \multirow{4}{*}{$6(48)$} & \multirow{2}{*}{ Threshold } & $75 \%$ vs $50 \%$ & $1.0(0.6)$ & .08 & $1.3(0.7)$ & .07 \\
\hline & & $100 \%$ vs $50 \%$ & $2.8(1.4)$ & $.04 *$ & $3.2(1.5)$ & $.04^{*}$ \\
\hline & \multirow{2}{*}{ Function } & Lin vs Exp & $1.1(1.0)$ & .28 & $1.2(1.0)$ & .26 \\
\hline & & Log vs Exp & $2.7(1.4)$ & $.03 *$ & $2.9(1.5)$ & $.01^{*}$ \\
\hline \multirow{4}{*}{$10(48)$} & \multirow{2}{*}{ Threshold } & $75 \%$ vs $50 \%$ & $2.2(0.7)$ & $<.001^{*}$ & $2.3(0.7)$ & $<.001^{*}$ \\
\hline & & $100 \%$ vs $50 \%$ & $4.2(1.2)$ & $<.001^{*}$ & $4.4(1.2)$ & $<.001^{*}$ \\
\hline & \multirow{2}{*}{ Function } & Lin vs Exp & $2.4(1.2)$ & $.04^{*}$ & $2.5(1.2)$ & $.04 *$ \\
\hline & & Log vs Exp & $6.3(1.5)$ & $<.001^{*}$ & $6.2(1.5)$ & $<.001 *$ \\
\hline \multirow{4}{*}{$16(48)$} & \multirow{2}{*}{ Threshold } & $75 \%$ vs $50 \%$ & $2.4(0.5)$ & $<.001^{*}$ & $2.3(0.5)$ & $<.001^{*}$ \\
\hline & & $100 \%$ vs $50 \%$ & $4.3(0.8)$ & $<.001^{*}$ & $3.8(0.8)$ & $<.001^{*}$ \\
\hline & \multirow{2}{*}{ Function } & Lin vs Exp & $3.1(1.1)$ & $.004^{*}$ & $2.8(1.1)$ & $.01^{*}$ \\
\hline & & Log vs Exp & $7.7(1.5)$ & $<.001^{*}$ & $6.8(1.6)$ & $<.001 *$ \\
\hline \multirow{4}{*}{24 (150) } & \multirow{2}{*}{ Threshold } & $75 \%$ vs $50 \%$ & $2.9(0.5)$ & $<.001^{*}$ & $2.5(0.5)$ & $<.001^{*}$ \\
\hline & & $100 \%$ vs $50 \%$ & $5.7(0.9)$ & $<.001^{*}$ & $4.8(0.8)$ & $<.001^{*}$ \\
\hline & \multirow{2}{*}{ Function } & Lin vs Exp & $4.3(1.2)$ & $<.001^{*}$ & $4.5(1.2)$ & $<.001^{*}$ \\
\hline & & Log vs Exp & $9.4(1.6)$ & $<.001^{*}$ & $9.2(1.6)$ & $<.001^{*}$ \\
\hline \multirow{4}{*}{$48(150)$} & \multirow{2}{*}{ Threshold } & $75 \%$ vs $50 \%$ & $2.3(0.3)$ & $<.001^{*}$ & $2.1(0.3)$ & $<.001^{*}$ \\
\hline & & $100 \%$ vs $50 \%$ & $4.5(0.5)$ & $<.001^{*}$ & $3.7(0.5)$ & $<.001 *$ \\
\hline & \multirow{2}{*}{ Function } & Lin vs Exp & $4.6(1.0)$ & $<.001^{*}$ & $3.9(0.9)$ & $<.001 *$ \\
\hline & & Log vs Exp & $8.3(1.5)$ & $<.001^{*}$ & $5.9(1.5)$ & $<.001^{*}$ \\
\hline
\end{tabular}

Table I. Direct contrasts showing the accuracy increase when using lower thresholds and exponential functions. To note, since the dependent variable in the mixed-effects models was the error in estimating original risk-taking profiles, which are expressed in a range [1 100], the estimates ( $\beta s$ ) in the table represent the percentage of improvement. For example, if the contrast " $100 \%$ vs $50 \%$ " is significant with a $\beta=4.3$, it means that using a maximum threshold of $50 \%$ increases the accuracy in reconstructing original risk-taking profiles by $4.3 \%$. As shown in the table, using lower thresholds and exponential functions generally increases accuracy independently on the maximum number of inflation events and the noise. 


\section{REFERENCES}

Ashenhurst, JR, Jentsch, JD, Ray, LA (2011). Risk-taking and alcohol use disorders symptomatology in a sample of problem drinkers. Experimental and Clinical Psychopharmacoology, 19(5), 361-370. Doi: 10.1037/a0024412

Bajracharya K, Duboz R (2013). Comparison of three agent-based platforms on the basis of a simple epidemiological model (WIP). In: Proceedings of the symposium on theory of modeling and simulation -DEVS integrative M\&S symposium, pp 6-11.

Bishara, AJ, Pleskac, TJ, Fridberg, DJ, Yechiam, E, Lucas, J, Busemeyer, JR, Finn, PR, Stout, JC (2009). Similar Processes Despite Divergent Behavior in Two Commonly Used Measures of Risky Decision Making. Journal of Behavioral Decision Making, 22(4), 435-454. Doi: 10.1002/bdm.641

Boka, E, Del Pozzo, J, Goetz, D, Remsen, B, Walsh-Messinger, J, Getz, M, Antonius, D, Malaspina, D (2020). Ineffective risk-reward learning in schizophrenia. Psychiatry Research, 293, 113370. https://doi.org/10.1016/i.psychres.2020.113370

Bornovalova, MA, Geadz, MA, Kahler, C, Aklin, WM, Lejuez, CM (2008). Sensation seeking and risk-taking propensity as mediators in the relationship between childhood abuse and HIV-related risk behavior. Child Abuse and Neglect, 32(1), 99-109. Doi: 10.1016/j.chiabu.2007.04.009

Brown, EC, Hck, SM, Gold, JM, Carpenter, WT, Fischer, BA, Prentice, KP, Wlatz, JA (2015). Integrating frequency and magnitude information in decision-making in schizophrenia: An account of patient performance on the lowa Gambling Task. Journal of Psychiatric Research, 66-67, 16-23. https://doi.org/10.1016/i.jpsychires.2015.04.007

Buelow, MT, Barnhart, WR (2017). The Influence of Math Anxiety, Math Performance, Worry, and Test Anxiety on the lowa Gambling Task and Balloon Analogue Risk Task. Assessment, 24(1), 127-137. Doi: 10.1177/1073191115602554

Canning, JR, Schallert, MR, Larimer, ME (2022). A Systematic Review of the Balloon Analogue Risk Task (BART) in Alcohol Research. Alcohol and Alcoholism, 57(1), 85-103. https://doi.org/10.1093/alcalc/agab004

Cazzell, M, Li, L, Lin, Z-J, Patel, SJ, Liu, H (2012). Comparison of neural correlates of risk decision making between genders: An exploratory fNIRS study of the Balloon Analogue Risk Task (BART). Neurolmage, 62, 1896-1911. Doi: 10.1016/j.neuroimage.2012.05.030

Chen, G, Pine, DS, Brotman, MA, Smith, AR, Cox, RW, Taylor, PA, Haller, SP (2022). Hyperbolic trade-off: The importance of balancing trial and subject sample sizes in neuroimaging. Neurolmage, 247, 118786. https://doi.org/10.1016/j.neuroimage.2021.118786

Cheng, GLF, Tang, JCY, Li, FWS, Lau, EYY, Lee, TMC (2012). Schizophrenia and risk-taking: Impaired reward but preserved punishment processing. Schizophrenia Research, 136(1-3), 122-127. https://doi.org/10.1016/i.schres.2012.01.002

Congdon, E, Bato, AA, Schonberg, T, Mumford, JA, Karlsgodt, KH, Sabb, FW, London, ED, Cannon, TD, Bilder, RM, Poldrack RA (2013). Differences in neural activation as a function of risk-taking task parameters. Frontiers in Neurosciences, 7, 173. Doi: 10.3389/fnins.2013.00173

Dean, AC, Sugar, CA, Hellemann, G, London, E. D. (2011). Is all risk bad? Young adult cigarette smokers fail to take adaptive risk in a laboratory decision-making test. Psychopharmacology, 215, 801-811. https://doi.org/10.1007/s00213-011-2182-y

De Groot, K, Thurik R (2018). Disentangling Risk and Uncertainty: When Risk-Taking Measures Are Not About Risk. Frontiers in Psychology, 9, 2194. Doi: 10.3389/fpsyg.2018.02194

De Groot, K, Strien, JW (2019). Event-related potentials in response to feedback following risk-taking in the hot version of the Columbia Card Task. Psychophysiology, 56, e13390. Doi: 10.1111/psyp.13390

De Groot, K (2020). Burst Beliefs - Methodological Problems in the Balloon Analogue Risk Task and Implications for Its Use. Journal of Trial and Error, 1(1), 1-10.

DeMartini, KS, Leeman, RF, Corbin, WR, Toll, BA, Fucito, LM, Lejuez, CW, O'Malley, SS (2014). A new look at risktaking: using a translational approach to examine risk-taking behavior on the balloon analogue risk task. Experimental and Clinical Psychopharmacology, 22(5), 444-452. Doi: 10.1037/a0037421 
Dominguez, DG (2011). The Relationship between Risk-Taking, Substance Abuse and Aggression in Schizophrenia. Doctoral Dissertation, Barnard College.

Donkin E, Dennis P, Ustalakov A, Warren J, Clare A (2017) Replicating complex agent based models, a formidable task. Environmental Modelling \& Software, 92:142-151

Fecteau, S, Pascual-Leone, A, Zald, DH, Liguori, P, Théoret, H, Boggio, PS, Fregni, F (2007). Activation of Prefrontal Cortex by Transcranial Direct Current Stimulation Reduces Appetite for Risk during Ambiguous Decision Making. Journal of Neuroscience, 27(23), 6212-6218. https://doi.org/10.1523/JNEUROSCI.0314-07.2007

Fernie, G, Cole, JC, Goudie, AJ, Field, M (2010). Risk-taking but not response inhibition or delay discounting predict alcohol consumption in social drinkers. Drug and Alcohol Dependence, 112(1-2), 54-61. https://doi.org/10.1016/j.drugalcdep.2010.05.011

Fischer, BA, McMahon, RP, Kelly, DL, Wehring, HJ, Meyer, WA, Feldman, S, Carpenter, WT, Gorelick, DA (2015). Risk-taking in schizophrenia and controls with and without cannabis dependence. Schizophrenia Research, 161(2-3), 471-477. https://doi.org/10.1016/j.schres.2014.11.009

Fitzpatrick, BG (2019). Issues in Reproducible Simulation Research. Bulletin of Mathematical Biology, 81(1), 1-6

Guenther, B, Galizzi, MM, Sanders, JG (2021). Heterogeneity in Risk-Taking During the COVID-19 Pandemic: Evidence From the UK Lockdown. Frontiers in Psychology, 12, 643653.

https://doi.org/10.3389/fpsyg.2021.643653

Guo, H, Zhang, Z, Da, S, Sheng, X, Zhang, X (2018). High-definition transcranial direct current stimulation (HD-tDCS) of left dorsolateral prefrontal cortex affects performance in Balloon Analogue Risk Task (BART). Brain and Behavior, 8, e00884. Doi: 10.1002/brb3.884

Hanson, KL, Thayer, RE, Tapert, SF (2014). Adolescent marijuana users have elevated risk-taking on the balloon analog risk task. Journal of Psychopharmacology, 28, 1080-1087. Doi: 10.1177/0269881114550352

Helfinstein, SM, Schonberg, T, Congdon, E, Karlsgodt, KH, Mumford, JA, Sabb, FW, Cannon, TD, London, ED, Bilder, RM, Poldrack, RA (2014). Predicting risky choices from brain activity patterns. PNAS, 111(7), 2470-2475. https://doi.org/10.1073/pnas.1321728111

Holt, DD, Green, L, Myerson, J (2003). Is discounting impulsive? Evidence from temporal and probability discounting in gambling and non-gambling college students. Behavioral Processes, 64, 355-367. Doi: 10.1016/S0376-6357(03)00141-4

Hopko, DR, Lejuez, CW, Daughters, SB, Aklin, WM, Osborne, A, Simmons, BL, Strong, DR (2006). Construct Validity of the Balloon Analogue Risk Task (BART): Relationship with MDMA Use by Inner-City Drug Users in Residential Treatment. Journal of Psychopathology and Behavioral Assessment, 28(2), 95-101. Doi: 10.1007/s10862-0067487-5

Hunt, MK, Hopko, DR, Bare, R, Lejuez, CW, Robinson, EV (2005). Construct validity of the Balloon Analog Risk Task (BART): Associations with psychopathy and impulsivity. Assessment, 12, 416-428. Doi: 10.1177/1073191105278740

Isles, AR, Winstanley, CA, Humby, T (2019). Risk taking and impulsive behaviour: fundamental discoveries, theoretical perspectives and clinical implications. Philosophical Transactions of the Royal Society: B, 374, 20180128. https://doi.org/10.1098/rstb.2018.0128

Kılıç, A, van Tilburg, WAP, Igou, ER (2020). Risk-taking increases under boredom. Journal of Behavior Decision Making, 33(3), 257-269.

King, KM, Patock-Peckam, JA, Dager, AD, Thimm, K, Gates, JR (2014). On the Mismeasurement of Impulsivity: Trait, Behavioral, and Neural Models in Alcohol Research among Adolescents and Young Adults. Alcohol, 1, 19-32. https://doi.org/10.1007/s40429-013-0005-4

Lawyer, SR (2013). Risk taking for sexual versus monetary outcomes using the Balloon Analogue Risk Task. The Psychological Record, 63, 803-820. Doi :10.11133/J.TPR.2013.63.4.007

Leigh, BC (1999). Peril, chance, and adventure: Concepts of risk, alcohol use and risky behavior in young adults. Addiction, 94, 371-383. Doi: 10.1046/j.1360-0443.1999.9433717.x 
Lejuez, CW, Read, JP, Whaler, CW, Richards, JB, Ramsey SE, Stuart, GL, Strong, DR, Brown, RA (2002). Evaluation of a Behavioral Measure of Risk Taking: The Balloon Analogue Risk Task (BART). Journal of Experimental Psychology: Applied, 8(2), 75-84

Lejuez, CW, Aklin, WM, Jones, HA, Richards, JB, Strong, DR, Kahler, CW, Read, JP (2003). The Balloon Analogue Risk Task (BART) differentiates smokers and nonsmokers. Experimental and Clinical Psychopharmacology, 11, 2633. Doi: 10.1037//1064-1297.11.1.26

Lejuez, CW, Simmons, BL, Aklin, WM, Daughters, SB, Dvir, S (2004). Risk-taking propensity and risky sexual behavior of individuals in residential substance use treatment. Addictive Behaviors, 29, 1643-1647. Doi: 10.1016/j.addbeh.2004.02.035

Lejuez, CW, Aklin, W, Bornovalova, M, Moolchan, E (2005). Differences in risk-taking propensity across inner-city adolescent ever- and never-smokers. Nicotine \& Tobacco Research, 7, 71-79. https://doi.org/10.1080/14622200412331328484

Lenhard, a, Lenhard, W, Gary, S (2019). Continuous norming of psychometric tests: A simulation study of parametric and semi-parametric approaches. PLoS One, 14(9), e0222279

Li, X, Pan, Y, Fang, Z, Lei, H, Zhang, X, Shi, H, Ma, N, Raine, P, Wetherill, R, Kim, JJ, Wan, Y, Rao, H (2020). Test-retest reliability of brain responses to risk-taking during the balloon analogue risk task. Neurolmage, 209, 116495. Doi: 10.1016/j.neuroimage.2019.116495

Long, EC, Kaneva, R, Vasilev, G, Moeller, FG, Vassileva, J (2020). Neurocognitive and Psychiatric Markers for Addiction: Common vs. Specific Endophenotypes for Heroin and Amphetamine Dependence. Current Topics in Medicinal Chemistry, 20(7), 585-597. Doi: 10.2174/1568026620666200131124608

Lu, Z-H, Chow, S-M, Ram, M, Cole, PM (2019). Zero-Inflated Regime-Switching Stochastic Differential Equation Models for Highly Unbalanced Multivariate, Multi-Subject Time-Series Data. Psychometrika, 84(2), 611-645

Luk, MSK, Chang, WC, Chong, CSY, Siu, CMW, Chan, SKW, Lee, EMH, Hui, CLM, Sun, YN, Lee, TMC, Lo, TL, Chen, EYH (2021). Altered risky decision making in patients with early non-affective psychosis. European Archives of Psychiatry \& Clinical Neuroscience, 271(4), 723-731. Doi: 10.1007/s00406-019-00994-2

Maner, JK, Richey, A, Cromer, K, Mallott, M, Lejuez, CW, Schmidt, NB (2007). Dispositional anxiety and riskavoidant decision-making. Personality and Individual Differences, 42, 665-675. Doi: 10.1016/j.paid.2006.08.016

Mishra, S, Lalumière, ML, Williams, RJ (2017). Gambling, risk-taking, and antisocial behavior: A replication study supporting the generality of deviance. Journal of Gambling Studies, 33, 15-36. Doi: 10.1007/s10899-016-96088

Nichols, T, Das, S, Eickoff, SB, Evans, AC, Glatard, T, Hanke, M, et al. (2017). Best practices in data analysis and sharing in neuroimaging using MRI. Nature Neuroscience, 20(3), 299-303. Doi: 10.1038/nn.4500

Pettorruso, M, Miuli, A, Di Natale, C, Montemitro, C, Zoratto, F, De RIsion, L, D’Andrea, G, Dannon, PN, Martinotti, G, Di Giannantonio, M (2021). Non-invasive brain stimulation targets and approaches to modulate gamblbingrelated decisions: A systematic review. Addictive Behaviors, 112, 106657. Doi: 10.1016/j.addbeh.2020.106657

Purcell, JR, Herms, EN, Morales, J, Hetrick, WP, Wisner, KM, Browkn, JW (2017). A review of risky decision-making in psychosis-spectrum disorders. Clinical Psychology Review, 91, 102112. https://doi.org/10.1016/i.cpr.2021.102112

Rao, H, Korczykowski, M, Pluta, J, Hoang, A, Detre, JA (2008). Neural correlates of voluntary and involuntary risk taking in the human brain: an fMRI Study of the Balloon Analog Risk Task (BART). Neurolmage, 42(2), 902-910. Doi: 10.1016/j.neuroimage.2008.05.046

Reddy, LF, Lee, J, Davis, MC, Altshuler, L, Glahn, DC, Miklowitz, DJ, Green, MF (2014). Impulsivity and Risk Taking in Bipolar Disorder and Schizophrenia. Neuropsychopharmacology, 39, 456-463. https://doi.org/10.1038/npp.2013.218

Rieser, NM, Shaul, L, Blankers, M, Koeter, MWJ, Schippers, GM, Goudriaan, AM (2019). The Predictive Value of Impulsivity and Risk-Taking Measures for Substance Use in Substance Dependent Offenders. Frontiers in Behavioral Neurosciences, 13, 192. Doi: 10.3389/fnbeh.2019.00192. 
Rinderknecht, MD, Lambercy, O, Gassert, R (2018). Enhancing simulations with intra-subject variability for improved psychophysical assessments. PLoS One, 13(12), 0209839

Robbins, TW, Gillan, CM, Smith, DG, de Wit, S, Ersche, TD (2012). Neurocognitive endophenotypes of impulsivity and compulsivity: towards dimensional psychiatry. Trends in Cognitive Sciences, 16, 81-91. Doi: 10.1016/j.tics.2011.11.009

Rose, AK, Jones, A, Clarke, N, Christiansen, P (2014). Alcohol-induced risk taking on the BART mediates alcohol priming. Psychopharmacology, 231, 2273-2280. Doi: 10.1007/s00213-013-3377-1

Schmitz, F, Manske, K, Preckel, F, Wilhelm, O (2016). The Multiple Faces of Risk-Taking: Scoring alternatives for the balloon-analogue risk task. European Journal of Psychological Assessment, 32(1), 17-38. DOI: 10.1027/1015$5759 / a 000335$

Schonberg, T, Fox, Cr, Poldrack, RA (2011). Mind the gap: bridging economic and naturalistic risk-taking with cognitive neuroscience. Trends in Cognitive Sciences, 15(1), 11-19. Doi: 10.1016/j.tics.2010.10.002

Sela, T, Kilim, A, Lavidor, M (2012). Transcranial alternating current stimulation increases risk-taking behavior in the Balloon Analog Risk Task. Frontiers in Neuroscience, 6, 1-11. Doi: 10.3389/fnins.2012.00022

Skeel, RL, Pilarski, C, Pytlak, K, Neudecker, J (2008). Personality and performance-based measures in the prediction of alcohol use. Psychology of Addictive Behaviors, 22(3), 402-409. Doi: 10.1037/0893-164X.22.3.402

Steiner, MD, Frey, R (2022). Representative design in psychological assessment: A case study using the Balloon Analogue Risk Task (BART). Journal of Experimental Psychology: General, 150(10), 2117-2136. Doi: 10.1037/xge0001036

Swogger, MT, Walsh, Z, Lejuez, CW, Kosson, DS (2010). Psychopathy and risk taking among jailed inmates. Criminal Justice and Behavior, 37, 439-452. https://doi.org/10.1177/0093854810361617

Tikàsz, A, Dumais, A, Lipp, O, Stip, E, Lalonde, P, Laurelli, M, Lungu, O, Potvin, S (2019). Reward-related decisionmaking in schizophrenia: A multimodal neuroimaging study. Psychiatry Research: Neuroimaging, 286(30), 4552. https://doi.org/10.1016/i.pscychresns.2019.03.007

Tonini, E, Quidé, Y, Kaur, M, Whitford, TJ, Green, MJ (2021). Structural and functional neural correlates of schizotypy: A systematic review. Psychological Bulletin, 147(8), 826-866. Doi: 10.1037/bul0000260

Turner, C, McClure, R, Pirozzo, S (2004). Injury and risk-taking behavior-a systematic review. Accident Analysis \& Prevention, 36(1), 93-101. Doi: 10.1016/s0001-4575(02)00131-8

Vassilva, J, Conrod, PJ (2019). Impulsivities and addictions: a multidimensional integrative framework informing assessment and interventions for substance use disorders. Philosophical Transactions of the Royal Society: B, 374, 20180137. Doi: 10.1098/rstb.2018.0137

Yakobi, O, Danckert, J (2021). Boredom proneness is associated with noisy decision-making, not risk-taking. Experimental Brain Research, 239, 1807-1825. https://doi.org/10.1007/s00221-021-06098-5

Weafer, J, Milich, R, Fillmore, MT (2011). Behavioral components of impulsivity predict alcohol consumption in adults with ADHD and healthy controls. Drug and Alcohol Dependence, 113(2-3), 139-146. Doi: 10.1016/j.drugalcdep.2010.07.027

White, TL, Lejuez, CW, de Wit, H (2008). Test-Retest characteristics of the Balloon Analouge Risk-Task (BART). Experimental and Clinical Psychopharmacology, 16(6), 565-570. Doi: 10.1037/a0014083

Xu, S, Pan, Y, Wang, Y, Spaeth, AM, Qu, Z, Rao, H (2016). Real and hypothetical monetary rewards modulate risk taking in the brain. Scientific Reports, 6, 29520. https://doi.org/10.1038/srep29520

Zickar, MJ (2020). Measurement Development and Evaluation. Annual Review of Organizational Psychology and Organizational Behavior, 7, 213-232 